\title{
DETECTION AND COUNTING OF ORCHARD TREES FROM VHR IMAGES USING A GEOMETRICAL-OPTICAL MODEL AND MARKED TEMPLATE MATCHING
}

\author{
Philippe Maillard ${ }^{\mathrm{a} *}$, Marília F. Gomes ${ }^{\mathrm{a}, \mathrm{b}}$ \\ ${ }^{a}$ UFMG, Departamento de Geografia, Av. Antônio Carlos, 6627, Belo Horizonte - MG, Brazil - philippe@ufmg.br \\ b INCRA, Av. Afonso Pena, 3500, Belo Horizonte - MG, Brazil - mariliafgomes@yahoo.com
}

Commission VII, WG VII/4-5

KEY WORDS: Orchards, VHR Images, Template Matching, Tree Crown Detection, Geometrical-Optical Model, Tree Counting

\begin{abstract}
:
This article presents an original algorithm created to detect and count trees in orchards using very high resolution images. The algorithm is based on an adaptation of the "template matching" image processing approach, in which the template is based on a "geometricaloptical" model created from a series of parameters, such as illumination angles, maximum and ambient radiance, and tree size specifications. The algorithm is tested on four images from different regions of the world and different crop types. These images all have $<1$ meter spatial resolution and were downloaded from the GoogleEarth application. Results show that the algorithm is very efficient at detecting and counting trees as long as their spectral and spatial characteristics are relatively constant. For walnut, mango and orange trees, the overall accuracy was clearly above $90 \%$. However, the overall success rate for apple trees fell under $75 \%$. It appears that the openness of the apple tree crown is most probably responsible for this poorer result. The algorithm is fully explained with a step-by-step description. At this stage, the algorithm still requires quite a bit of user interaction. The automatic determination of most of the required parameters is under development.
\end{abstract}

\section{INTRODUCTION}

It is estimated that orchard fruit production represents approximately $3 \%-4 \%$ of the total arable land (FAO, 2012). The EU CAP regulations (EC 73/2009) provide support for permanent crops like hazelnuts, almonds, walnuts and fruits in general (Santoro et al., 2013; Aksoy et al., 2012; Tasdemir, 2010) but eligible orchards need to have a certain size and tree density, depending on the type of crop. The task of estimating fruit production generally implies counting trees, a time consuming task that could benefit from the use of the very high resolution (VHR) satellite images now available, but also the near-global high resolution image coverage such as is provided by Google-Earth and other internet-based image services. An orchards is a plantation of trees of the same species and often the same age. Consequently, trees in orchards usually have similar sizes and shapes and are regularly spaced. Image processing can be adapted to such a task, providing efficient algorithms that can take advantage of the particular aspect of the orchards and the availability of VHR images.

Very high spatial resolution remote sensing images represent a cost effective and reliable way of obtaining information about trees. It may be the only practical manner to ensure a sustainable stewardship of forests with the necessary data about trees in a synoptic and repetitive form for large areas and over long periods of time (Shao and Reynolds, 2006). As remote sensing goes, it is generally the tree crown that stands as the basis for the data required for the inventory, for it allows determining not only the tree's size but also its position, the density of leaf coverage, and in some cases the species. It can also be used to estimate ecological parameters such as the density of the population, the health condition of the trees, their volume, their biomass, and carbon sequestration rates (Ke and Quackenbush, 2011; Perrin, 2006; Erickson, 2004).

The analysis of individual trees based on remote sensing images is a complex problem given the variability of the size, shape, and

\footnotetext{
${ }^{*}$ Corresponding author
}

spectral response of the crown. What is detected as a single object may in fact be, in some cases, a separate group of branches, or in other cases a group of trees(Pu and Landry, 2012). Other sources of error are caused by the proximity of neighbouring trees, trees' being located under other trees, trees in the shade, or trees that have a low spectral contrast with the background (Wulder et al., 2000). Still, the information provided this way is crucial to a series of applications, such as the inventory and management of forested areas as well as of parks and urban forests. It can also be used for counting and monitoring trees in orchards or under power lines to prevent damage and accidents. In the case of orchards, VHR remote sensing can also contribute to early stress detection, yield prediction, and phenology monitoring (Stuckens et al., 2010).

Larsen et al. (2011) have made a review of six algorithms (arguably the most well recognized) for the detection of individual trees and concluded that no single algorithm is best for all situations of tree density and characteristics. Furthermore they found template matching (TM) to produce the best results for individual isolated trees, which is often (but not always) the situation in orchards where trees are generally well separated from each other.

Recently, Gomes and Maillard (2014) used a marked point-process (MPP) and TM with a 3D geometrical-optical model (GOM) to locate isolated trees of varying size and shape in urban environments. Because trees in orchards are often individually distinguishable and have a similar shape and size, they are perfect candidates for TM with a 3D GOM. In this article, VHR Google Earth images were used for identifying orchard trees. Orchards being collections of individual trees often arranged regularly, a combined TM-GOM algorithm can easily be adapted for the task of tree counting in a timely fashion.

A number of approaches have been proposed for the mapping of orchards using VHR images, some of which are presented in Table 1. All these approaches use some primitive structur- 
ing element which is compared to the image or texture analysis or a combination of both. The use of some structuring element (usually a circular shape) is closely related to template matching (TM).

In this selection of approaches, three (Bazi et al., 2009; Recio et al., 2013; Santoro et al., 2013) used some morphological approach based on some simple shape to detect the trees. A fourth (González et al., 2007) used a Chamfer transform, which is also shape-based. One approach employed region growing on spectral features (Srestasathiern and Rakwatin, 2014) and two (Tasdemir, 2010; Aksoy et al., 2012) aimed at detecting whole orchard plots rather than the trees. These latter two approaches offer an interesting complement to the tree detection problem, in which the detection of the orchards prior to the tree detection phase would reduce significantly the search areas and eliminate false positives found outside the orchards.

It has become clear that the use of some kind of morphological element represents a key element in detecting orchard trees in VHR images. It is also the approach we have favoured here but we opted to introduce a three-dimensional structuring element based on a GOM to make the approach more consistent with the specific characteristics of the image at hand.

\begin{tabular}{|c|c|c|}
\hline Reference & Algorithm & Comments \\
\hline $\begin{array}{l}\text { González et } \\
\text { al. (2007) }\end{array}$ & $\begin{array}{l}\text { Chamfer } \\
\text { transform }\end{array}$ & $\begin{array}{l}\text { Comparison with flat dark disk } \\
\text { and probability of being in a } \\
\text { reticular arrangement. }\end{array}$ \\
\hline $\begin{array}{l}\text { Tasdemir } \\
\text { (2010) }\end{array}$ & $\begin{array}{l}\text { Self- } \\
\text { organizing } \\
\text { map }\end{array}$ & $\begin{array}{l}\text { Combining spectral and tex- } \\
\text { ture features to detect orchards } \\
\text { as whole objects }\end{array}$ \\
\hline $\begin{array}{l}\text { Aksoy et al. } \\
(2012)\end{array}$ & $\begin{array}{l}\text { Texture- } \\
\text { based } \\
\text { classifica- } \\
\text { tion }\end{array}$ & $\begin{array}{l}\text { Texture based on primitive and } \\
\text { granulometric analysis using } \\
\text { Gabor filters. Pre-processing } \\
\text { by applying a Laplace of Gaus- } \\
\text { sian filter. }\end{array}$ \\
\hline $\begin{array}{l}\text { Santoro et } \\
\text { al. }(2013)\end{array}$ & $\begin{array}{l}\text { Structuring } \\
\text { elements } \\
\text { and classifi- } \\
\text { cation }\end{array}$ & $\begin{array}{l}\text { Uses an asymmetrical filter to } \\
\text { account for differential illumi- } \\
\text { nation. Non-vegetation areas } \\
\text { are masked using NDVI. }\end{array}$ \\
\hline $\begin{array}{l}\text { Recio et al. } \\
\text { (2013) }\end{array}$ & $\begin{array}{l}\text { Morphological } \\
\text { operator on } \\
\text { classified } \\
\text { image }\end{array}$ & $\begin{array}{l}\text { Uses unsupervised } k \text {-means, } \\
\text { detects tree class, and ap- } \\
\text { plies morphological operator } \\
\text { to eliminate false positives }\end{array}$ \\
\hline $\begin{array}{l}\text { Bazi et al. } \\
(2009)\end{array}$ & $\begin{array}{l}\text { Morphological } \\
\text { operator on } \\
\text { binary } \\
\text { image }\end{array}$ & $\begin{array}{l}\text { First classifies image, then } \\
\text { uses morphological analysis } \\
\text { (erosion). }\end{array}$ \\
\hline $\begin{array}{l}\text { Srestasathiern } \\
\text { and Rak- } \\
\text { watin } \\
(2014)\end{array}$ & $\begin{array}{l}\text { Local peak } \\
\text { detection }\end{array}$ & $\begin{array}{l}\text { Uses a series of spectral fea- } \\
\text { tures and rank filtering. Cal- } \\
\text { culates window size based on } \\
\text { semi-variogram. }\end{array}$ \\
\hline
\end{tabular}

Table 1: List of articles dedicated to orchard tree detection using VHR images $(\leq 1 \mathrm{~m})$ along with their respective generic approach.

The objective of this research is to introduce an adaptation of TM with a 3D GOM to detect and count trees in orchards in VHR images such as provided by Google Earth. The TM is adapted to this particular situation by placing marks to "reserve" the space for each tree found. The approach also incorporates a module to determine a near-best model (GOM radius, and illumination parameters) from an image sample. Lastly, the algorithm can handle both monochromatic and multi-spectral images. Results are presented for four sample scenes (two monochromatic and two multi-spectral) from different countries and crop types.

\section{METHOD}

By using a GOM, the effects of varying illumination (sun elevation and azimuth) become an advantage rather than an obstacle, especially when the background is homogeneous. In terms of data, VHR image data, including a large proportion of Google Earth images, have sufficient resolution for identifying orchard trees. In this case however, the illumination parameters are not readily available and must be determined. Although we have implemented an automated routine to determine most of these parameters (sun elevation, sun azimuth, crown size), it is still in development to make it perform better and the user's visual estimation is usually quicker and often better than our automated estimation.

The proposed approach is to introduce an adaptation of the algorithm mentioned above (TM and MPP) to detect and count trees in orchards of different types. Aiming at a regional application, Google Earth images were used to demonstrate a proofof-concept with images of lesser spectral and radiometric quality. The algorithm uses measure of the similarity between the GOM and the image to estimate the probability of being the centre of a tree and then places a mark for that tree in a non-overlapping position (unless some overlapping is allowed). The overlapping parameter can also be used to force a minimum spacing between the tree crowns; in this case a negative value is used. The algorithm also incorporates a module to determine the illumination parameters (sun azimuth and elevation, maximum and ambient radiance) from a sample.

\subsection{Geometrical-Optical 3D Model}

In creating a valid 3D geometrical-optical model we have chosen a simplified version in which the crown is represented by a dome of varying skewness, a Lambertian reflectance model with ambient light and a projected shadow on the ground. Equations 1 and 2 give the formulation of our model, in which each pixel is treated as a singular surface.

$$
L=L_{\max } \times\left(\frac{\cos (\theta)}{\cos (i)}\right)+a m b
$$

where

$\cos (i)=\left\{\cos \left(\theta_{s}\right) \cos \left(\theta_{n}\right)\right\}+\left\{\sin \left(\theta_{s}\right) \sin \left(\theta_{n}\right) \cos \left(\phi_{s}-\phi_{n}\right)\right\}$

Here, $L$ is the radiance of the pixel, $L_{\max }$ is the maximum radiance of the tree, $\cos (i)$ is the angle between the incidence angle of the sun and the normal of the tree surface, $\theta_{s}$ is the solar elevation angle, $\theta_{n}$ is the slope of the object surface, $\phi_{s}$ is the solar azimuth, $\phi_{n}$ is the aspect of the object surface and $a m b$ represents the ambient diffuse lighting.

The geometrical-optical model is adjusted according to the specific illumination parameters of the image and the size of the trees present on the scene. Figure 1 shows two examples of tree models with similar reflectances but different solar elevations.

A parameter of projected shadow clipping has also been added to take into account the fact that it was not always beneficial to use the whole shadow in situations where it was projected onto another tree and not on the ground. The height of the tree also affects the size of the shadow, so that it did not appear wise to set the height to a fixed value: it was made proportional to the crown radius. 


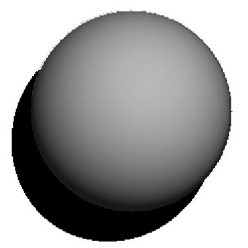

(a)

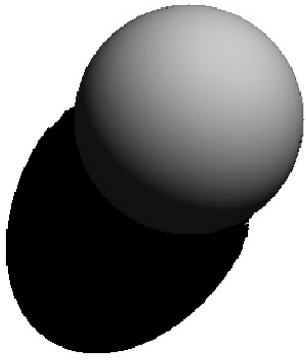

(b)
Figure 1: Illustration of the geometrical-optical model of tree crown as seen in two different solar elevation angles: (a) $20^{\circ}$ and (b) $45^{\circ}$.

\subsection{Algorithm}

The algorithm is based on three principles: Firstly, it assumes that the trees have a dome-like shape approximated with a GOM and the right illumination parameters; Secondly, that there is little or no overlap between the trees; Thirdly, that the pixel with the highest similarity represents the most likely central position of the tree.

The algorithm is not based on a "marked point process" since it is not completely random. It does however take advantage of the principle of a mark to ensure that the trees found follow a rank based on the similarity between the image and the GOM. The marked process makes sure that the location where a tree has been detected cannot be occupied by another tree unless some overlap has been previously allowed. The GOM is a simple dome model in which the height is estimated to be 1.5 times the diameter of the crown. To simplify the problem, we have employed the same diameter for all trees in the orchard (this can eventually be modified to incorporate a range of diameters). The algorithm responsible for the detection of trees can be best explained through a step-by-step description.

Step 1. Get user parameters: percentage of overlap allowed, minimum similarity value, tree diameter, and coordinate of sample tree. These parameters cannot yet be estimated automatically and are entered by the user. The illumination parameters can optionally be entered by the user; if not, they will be estimated by the program using the tree sample.

Step 2. If the sun elevation and azimuth are not provided by the user, these parameters are automatically estimated by the program using the coordinates of at least one tree sample from the image. The program then computes the similarity between the sample and all possibilities of illumination parameters in steps of 10 degrees in azimuth and 5 degrees in sun elevation.

Step 3. Calculate a similarity value (SV) for each pixel. The similarity is a combination of the cross-correlation and the normalized absolute difference between the GOM and the image at each pixel location and is given by Eq. 3 .

$$
S m=\gamma-\alpha N D
$$

where $\gamma$ is the cross-correlation, $N D$ is the normalized absolute difference, and $\alpha$ is a constant weight factor (usually between 0.25 and 0.5 depending on radiometric quality of the image). The cross-correlation and absolute difference are calculated as follows (Eq. 4 and 5).

$$
\gamma(x, y)=\frac{\sum_{i} \sum_{j}\left[\boldsymbol{\omega}_{(i, j)}-\overline{\boldsymbol{\omega}}\right] \sum_{i} \sum_{j}\left[\boldsymbol{f}_{(i, j)}-\overline{\boldsymbol{f}}\right]}{\left\{\sum_{i} \sum_{j}\left[\boldsymbol{\omega}_{(i, j)}-\overline{\boldsymbol{\omega}}\right]^{2} \sum_{i} \sum_{j}\left[\boldsymbol{f}_{(i, j)}-\overline{\boldsymbol{f}}\right]^{2}\right\}^{1 / 2}}
$$

Here, $\boldsymbol{\omega}_{i, j}$ is the GOM and $\boldsymbol{f}_{i, j}$ is the portion of the image that corresponds to a circle of the same radius, so that two matrices of same dimensions are always compared. The $\overline{\boldsymbol{\omega}}$ and $\overline{\boldsymbol{f}}$ are their respective means. The values of $\gamma$ range between -1 and 1 . The same logic is used in Eq. 5, which computes a normalized difference value between the same two matrices.

$$
N D=\left|\frac{\sum \boldsymbol{\omega}-\sum \boldsymbol{f}}{\sum \boldsymbol{\omega}+\sum \boldsymbol{f}}\right|
$$

Step 4. If the image is a multispectral image, repeat step 3 for all bands and make the similarity cumulative (the final value will then be divided by the number of channels/bands).

Step 5. Sort the pixels by decreasing similarity and store the coordinates. If the value is lower than the minimum allowed, the pixel is not stored.

Step 6. Place a temporary tree mark (circle) at the next pixel location with the highest similarity value.

Step 7. Verify whether the space is already occupied by a tree. If some overlap is allowed, make sure that the number of non-zero pixels is smaller than the percentage of overlap allowed. An output image is created to receive a permanent mark of the GOM shape with the associated similarity value.

Step 8. Validate the results. The validation is performed by comparing the number of trees found and their locations. The comparison is made by tree and not by pixel. Alternatively, it can be done by estimating the overall number of trees using the density of a representative sample and comparing this with the number of trees found.

By ordering the image pixels by their SV and progressively occupying the space with tree marks, the tree detection criterion automatically decreases with time until it reaches a minimum specified by the user.

\subsection{Test and Validation}

To illustrate the adapted TM-GOM algorithm we have tested it on four different types of orchards: a mango plantation in Brazil near Juazeiro (BA), a walnut plantation in France near Grenoble, an apple orchard near Southington (CT), and an orange plantation near Citrus Grove (FL) - the last two in the United States. These images were selected more or less arbitrarily using the four fruits as criterion and searching for regions renowned for their production. These regions are from both temperate (Grenoble and Southington) and tropical (Citrus Grove and Juazeiro) environments. The four images were directly extracted from Google Earth and had a relatively bad quality, as they appear to have been 
enhanced for sharpness. The first two were used in monochromatic mode while the last two were kept in RGB mode. To validate the results, we asked three geography students to manually interpret and mark the trees belonging to the orchards for all test images and we have evaluated the results in the following way:

- the total number of trees $(\mathrm{N})$ was determined by the interpreters;

- matching trees were computed as true positives (TP) and are defined by the number of trees found by the algorithm;

- unmatched trees (present on the image but absent from the results) were marked as false negatives (FN);

- trees marked by the algorithm but not by the interpreters were marked as false positives (FP);

- the final accuracy was computed as $\frac{T P}{N+F N} \times 100$.

The results show the proportion of true tree crowns found relative to the total number of tree crowns that should have been found plus the crowns that were found by the algorithm but are not trees. By further adding the wrongly found trees, the index shows a fairer value of success.

The interpreters were told not to mark trees that were much smaller or larger than the average tree in the orchards. This was initially necessary because we used a single crown size to compute similarity values in our tests. We are currently correcting this shortcoming by applying successive passes with different crown sizes, starting with larger crown sizes and ending with smaller ones. This order is logical since larger trees occupy larger areas and can be more difficult to find, whereas small tree GOMs can produce high similarity values even on large trees but not the inverse. Also, valid trees that were found by the algorithm but did not pertain to an orchard were not computed as false positives. As a further improvement, restricting the search to within the boundaries of the orchards would increase the accuracy and would enable the similarity parameter to be relaxed.

\section{RESULTS AND DISCUSSION}

Table 2 shows an overview of the results for the four test images while the graphical results are shown in Figures 2 and 3. Figure 2 shows the original images and a colour-coded result where the warmer tones indicate stronger similarity values and Figure 3 represents a mapping of the results showing the true positives (o), the false negatives or missing trees $(\bullet)$, and the false positives $(\times)$. Figure 4 shows the histogram of similarity values for the four images as well as the sample tree used to compute the maximum relative radiance and the ambient light (the relative radiance in the shadows).

The Grenoble test image (Figures 2a and 3a) is characterized by densely arranged walnut trees which have a large round crown (Figure 5a) so that the model was well correlated with the trees in the image and produced an overall accuracy of $\approx 90 \%$. But the fact that the trees are close to one another still produced 103 "misses". This forced the relaxation of the similarity threshold and caused a few false positives (69), all of which are outside the orchards. Interestingly, the histogram of SVs (Figure 4a) is clearly bimodal with the right peak roughly corresponding to the walnut trees.

In the case of Juazeiro (Figures $2 b$ and $3 b$ ), the area is populated by mango trees that, like the walnut trees, have large round
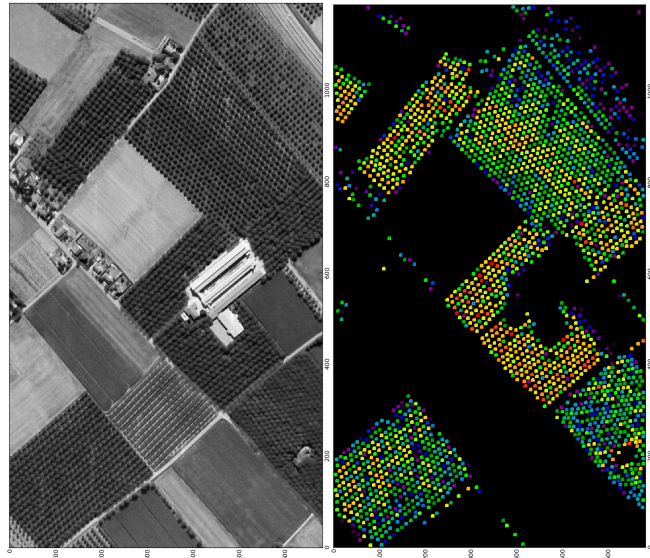

(a)
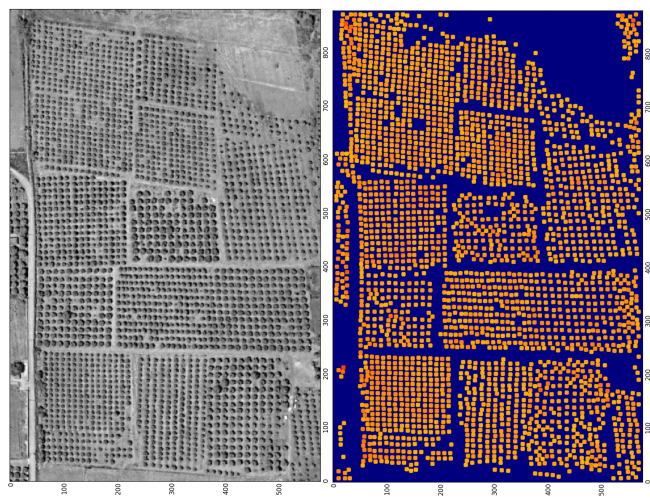

(b)
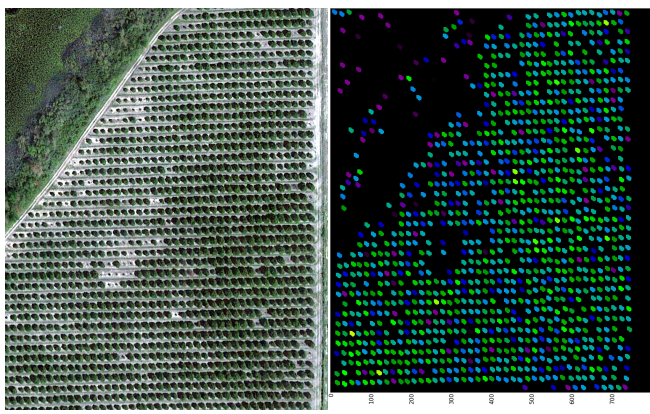

(c)
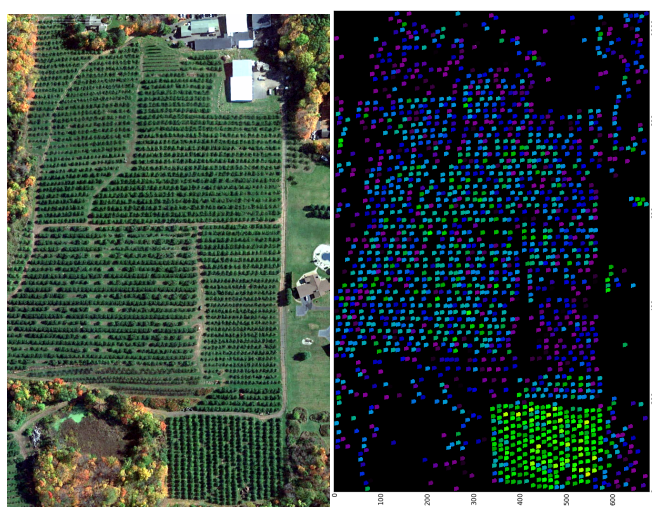

(d)

Figure 2: Results of the tree counting algorithm for the four test images: a) Grenoble, b) Juazeiro, c) Citrus Grove, d) Southington. The left column shows the original images while the right one shows the colour-coded results where warmer colours represent higher similarity values. 


\begin{tabular}{ll|rrrrrc}
\hline \hline Test Image & $\begin{array}{l}\text { Type } \\
\text { M/C }\end{array}$ & N & TP & FP & FN & $\begin{array}{c}\text { Overall } \\
\text { accuracy }\end{array}$ & $\begin{array}{c}\text { User } \\
\text { accuracy }\end{array}$ \\
\hline Grenoble & M & 2435 & 2289 & 69 & 103 & $90.19 \%$ & $94.00 \%$ \\
Juazeiro & M & 2534 & 2441 & 114 & 93 & $92.92 \%$ & $96.33 \%$ \\
Citrus Grove & $\mathrm{C}$ & 1185 & 1131 & 39 & 54 & $91.28 \%$ & $95.44 \%$ \\
Southington & $\mathrm{C}$ & 1873 & 1576 & 224 & 297 & $72.63 \%$ & $84.14 \%$ \\
\hline \hline
\end{tabular}

Table 2: Results of the tree counting algorithm for the four test images (France, Brazil, two in the USA). Legend: Type $\mathrm{M}=$ monochromatic, Type $\mathrm{C}=$ color $\mathrm{N}=$ number of trees, $\mathrm{TP}=$ true positive, $\mathrm{FP}=$ false positive, $\mathrm{FN}=$ false negative. User accuracy is the success rate attained when considering only the trees within the orchard's limits (no comission error).

crowns (Figure $5 \mathrm{~b}$ ) that generated a success rate of $\approx 93 \%$. The algorithm produced a fair amount of both false positives (114) and false negatives (93) mainly because of the variation of tree crown size and the particular situation of the dirt road on the left of the image that created a pattern of light and shade similar to the trees (approximately one-third of the false positives came from that road). Unlike the Grenoble case, the histogram of SVs (Figure $4 \mathrm{~b}$ ) has a unique peak showing that there is little difference between the trees and their surroundings. Again, all false positives are outside the orchards.

The Citrus Grove colour test image generated somewhat similar results with $\approx 91 \%$ overall success (Figures $2 \mathrm{c}$ and $3 \mathrm{c}$ ). The fact that colour was used improved the results by about $6 \%$ in relation to using a monochromatic channel (not shown here). Although most trees in the orchards are well defined, there are quite a few clearly smaller trees that were not detected. The SV histogram is clearly multi-modal with three dominant peaks and some secondary ones. This is due to the effect of using the three colour channels since some pixels were correlated to the GOM in one channel but not in the other ones, and shows the contribution of colour to help separate trees that are similar to the GOM and the sample relative radiance values.

The Southington test image produced poorer results with an overall success rate of only $\approx 73 \%$ (Figures $2 \mathrm{~d}$ and $3 \mathrm{~d}$ ). We attribute this to two main facts. Firstly, apple trees are known to be illshaped, with a sparser crown than the other three fruit trees (Figure 5d). Secondly, the orchards in Figure 3d are not very regular, with trees varying widely in size and spacing. The interpreters reported having the most difficulty with the Southington test image because of these variations. It can be observed in the two figures (Figures 2d and 3d) that the orchard located on the Southeast corner was well processed with only one false negative. This orchard is also characterized by the warmest colour, meaning that high SV were obtained in it.

The colour-coded images based on SV (Figure 2, right column) give a good indication of the strengths and weaknesses of the algorithm. As shown in Table 2, the algorithm worked well in most situations. It did not perform well in cases where the trees were much smaller or much bigger than the average or when their shape was very different from the idealized dome we used as a GOM. Similarly, it did not produce high SV in cases where the tree crowns overlap each other, as in the Southeast orchard of the Grenoble image (Figure 2a). It also appears that the edges of the orchards generated more false negatives, probably because these edges are often in contact with other objects like trails, roads, houses or even trees of different species with a different size. The most common of these problems are illustrated in Figure 6. A significant improvement could be achieved by limiting the search to within the orchards' boundaries, which would eliminate most false positives, as shown in the rightmost column of Table 2 (user accuracy).

In its current stage of development, the algorithm requires eight parameters: sun elevation, sun azimuth, maximum radiance, ambient radiance, crown radius, percent of projected shadow (GOM), minimum similarity value, and spacing/superposition allowed. The first six parameters can be estimated through a routine that is activated when the parameter is not supplied. For this to take place, the user needs to provide at least one coordinate of a typical tree crown in the image (if more than one coordinate is supplied, the routine analyses all trees and returns average values for the six parameters). At this stage we have found that a visual estimation of these parameters by the users is at least as good as the one supplied by the routine, and we are still working on improving the parameter estimation routine. In the case of real panchromatic or multi-spectral images, the first two parameters are included in the metadata of the image. Our next version of the algorithm will also include the possibility of running successive passes each with a different tree crown size, starting with the larger radius.

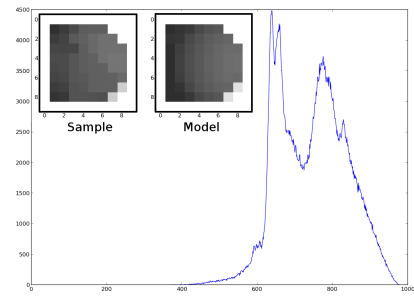

(a) Grenoble

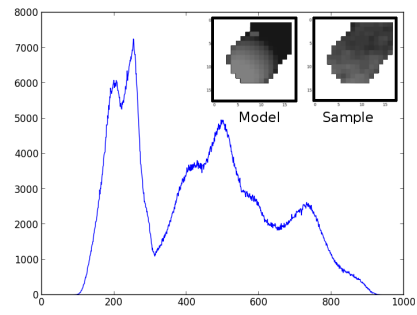

(c) Citrus Grove

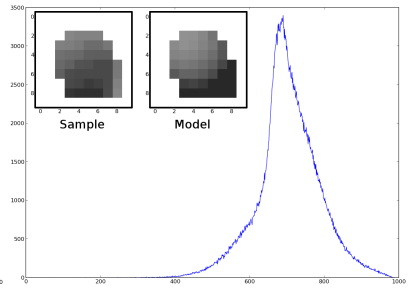

(b) Juazeiro
Figure 4: Similarity value histograms for the four test images. The grey tone corner images represent the the scale geometricaloptical models and the sample on which it was based.

\section{CONCLUSIONS}

Orchards have important VHR image features that can take advantage of crown recognition algorithms to ease the tasks of counting and monitoring trees. In this article we presented an adaptation of Template Matching that uses a geometrical-optical tree model based on illumination and radiance parameters. The algorithm also features a search by rank based on similarity values and the placement of marks to preserve the spatial distribution of the trees within the orchards. The algorithm can use both monochromatic or multi-spectral images and was tested in four different contexts with four different types of tree: walnut, mango, orange and apple. These test images were not original images but processed ones directly downloaded from the GoogleEarth web application. It was found that the first three types produced results above $90 \%$ but the apple orchards generated much poorer results, mostly because the apple tree is not as well shaped as the other three types. The program incorporates a module to automatically determine the illumination and radiance parameters. The illumination parameters are, however often better determined visually 


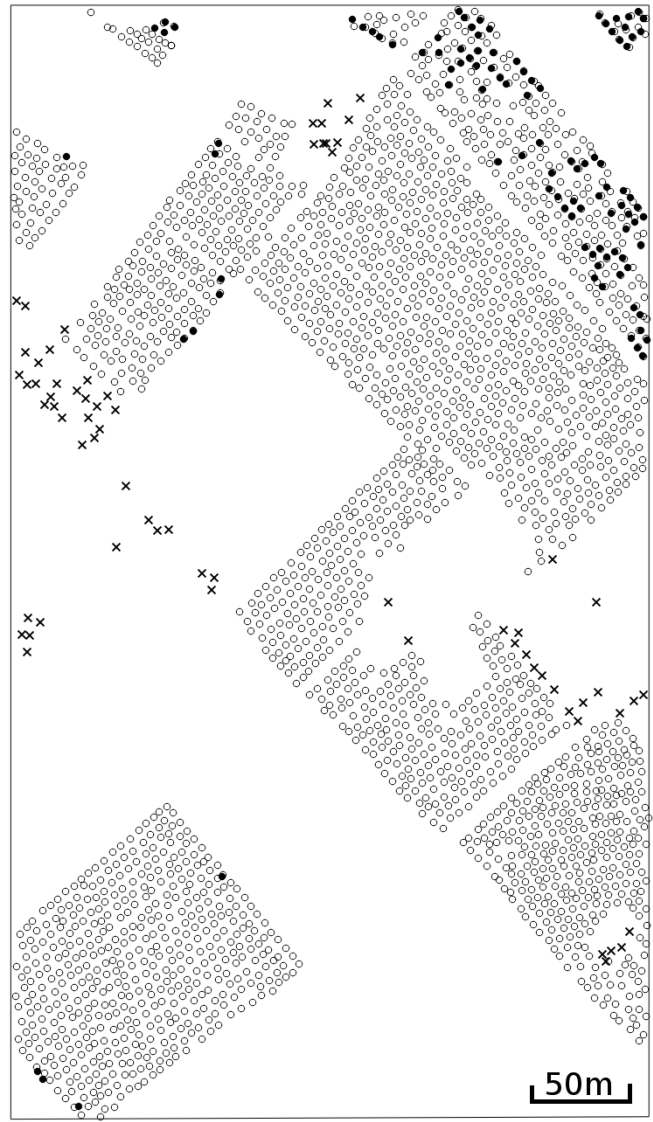

(a)

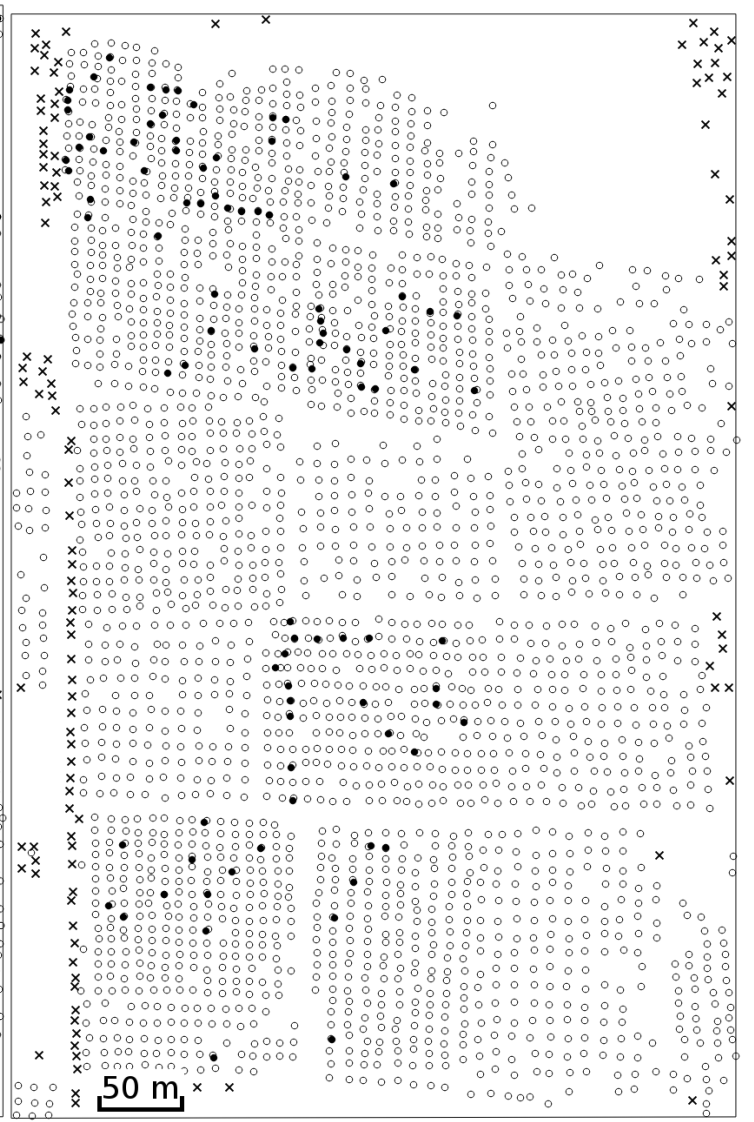

(b)

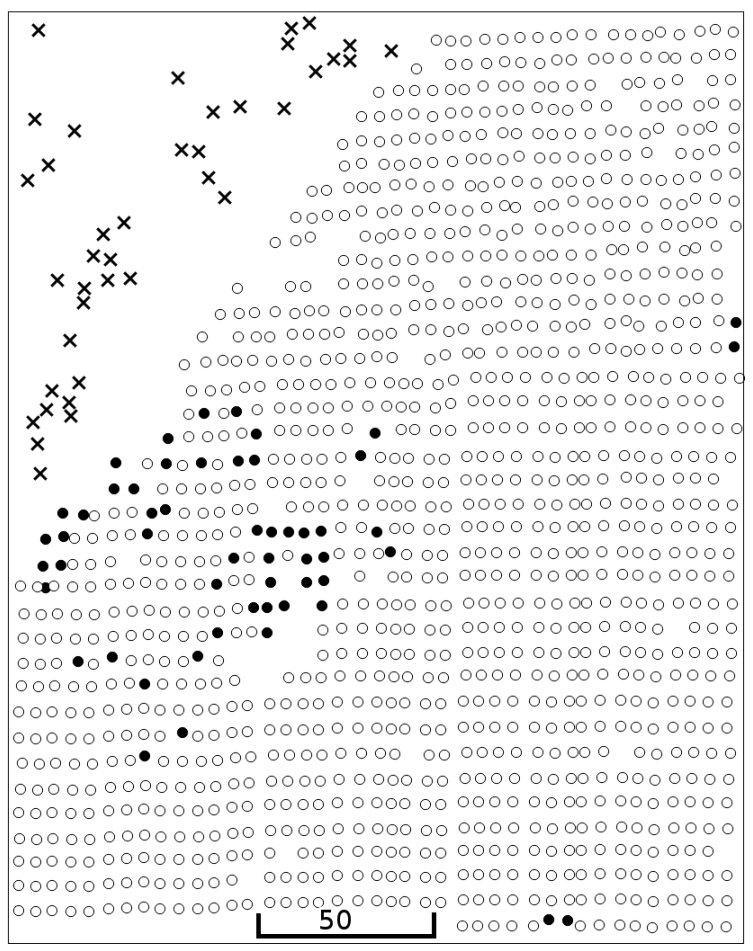

(c)

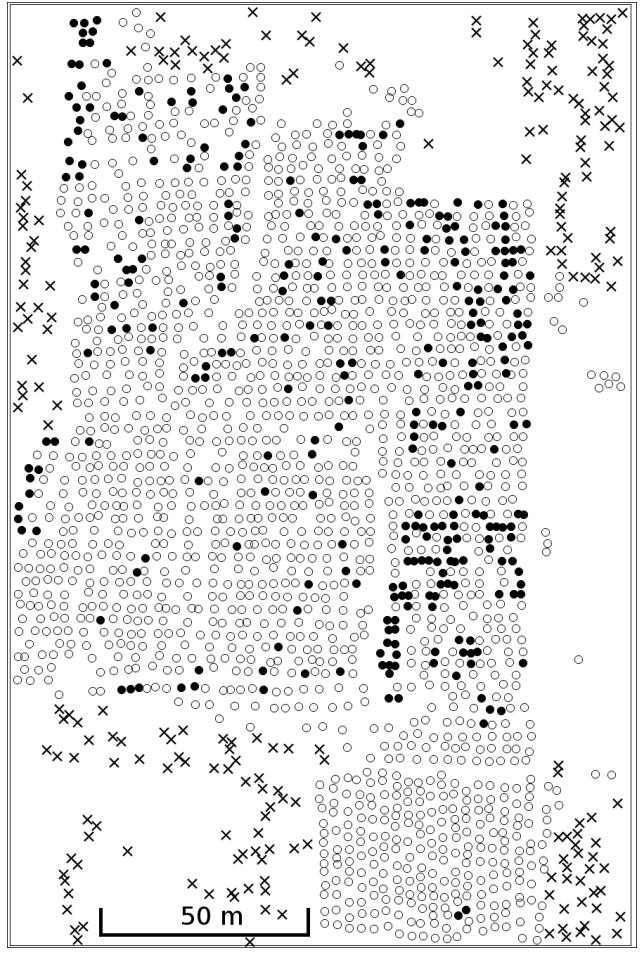

(d)

Figure 3: Mapping of the tree counting results for the four test images (a) Grenoble, b) Juazeiro, c) Citrus Grove, d) Southington): empty circles ( $(\circ)$ represent trees that were found, " $\times$ "s represents the false negatives and the black circles $(\bullet)$ represent the false positives. 


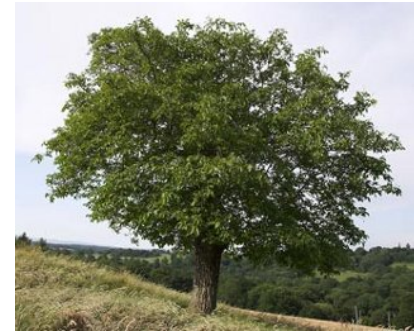

(a) Walnut

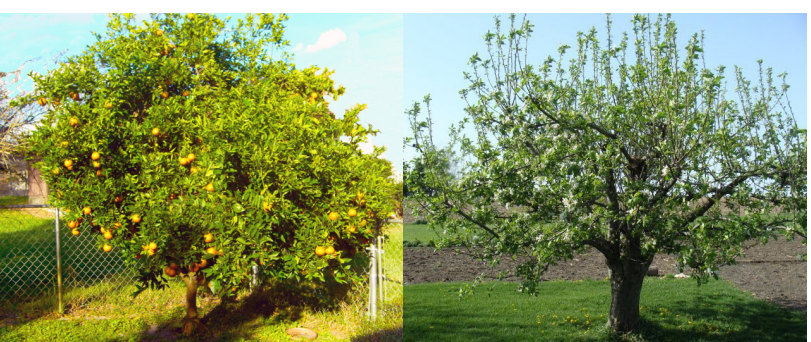

(c) Orange

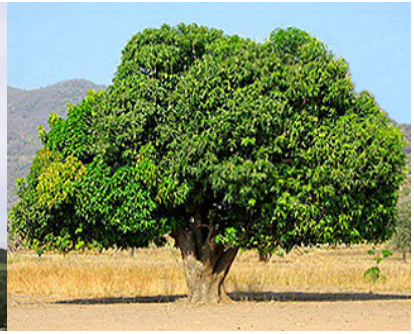

(b) Mango

(d) Apple
Figure 5: The different types of fruit trees analysed with the treecounting algorithm. Note how the apple tree crown is much more open than the other three types.
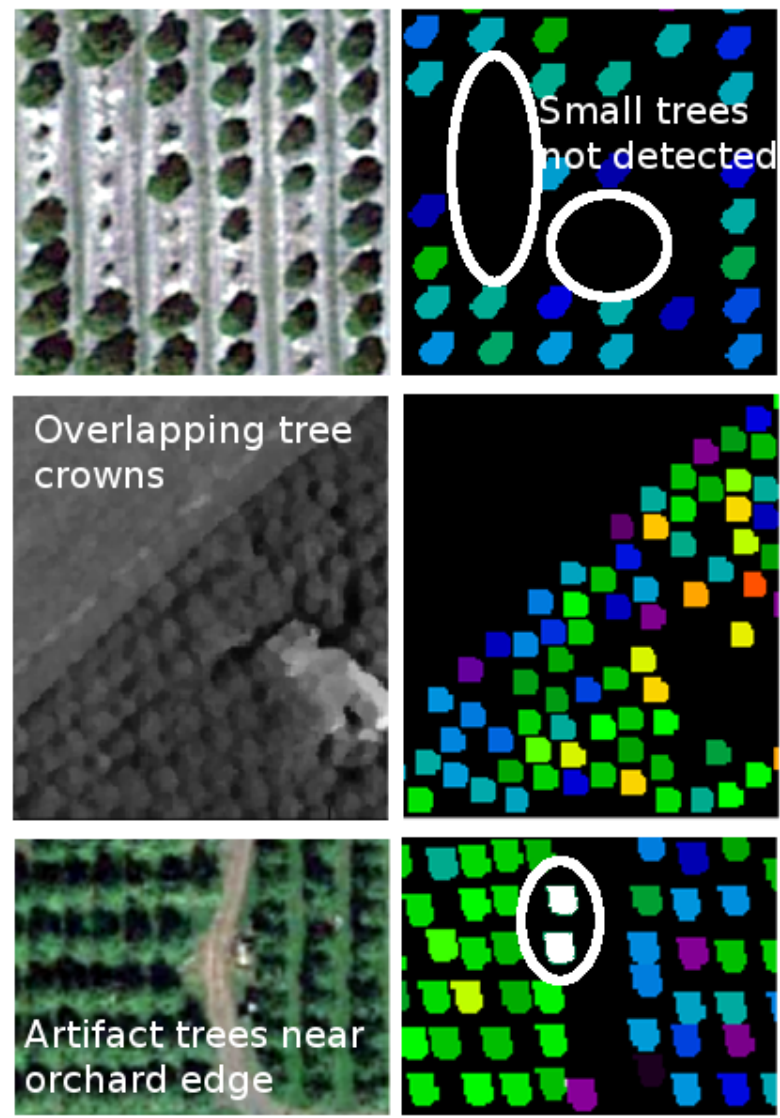

Figure 6: Some of the problem situations encountered by the tree counting algorithm.

by the user. The following points summarize the results obtained.

- Almost all trees found within the orchards' limits are true positives.
- Without considering the false positives outside the orchards' limits, the overall success increases to over $93 \%$ in three of the four test images (except Southington).

- Many false negatives created doubts in the interpreters, because of their small size or their odd shape.

- The algorithm is quite efficient when the trees in the orchards are well separated and have a uniform shape.

In its present state, the algorithm still relies on a threshold that is set by the user, but we think this could be replaced by limiting the search to actual orchards and providing a tree density value. Further refinements will test other measures of similarity, like the Spectral Information Divergence (SID) proposed by Du et al. (2004) and used by Stuckens et al. (2010) for citrus tree recognition. Work is also underway to incorporate the context and take advantage of the fact that orchard trees are never isolated. Lastly, we are looking into testing the approach proposed by Tasdemir (2010) that detects orchards as a single object before detecting and counting the tree crowns within the orchards, which would lead to a more robust hierarchical approach.

\section{ACKNOWLEDGEMENTS}

This work was produced within the context of a project of tree crown detection of the authors, and did not receive any specific financing. The authors are grateful to Google for enabling the download of very high resolution colour images from the Google Earth application. Finally we would like to thank Barbara Henrique Oliveira, Iara Marques Vilela and Dayane Freitas for mapping all the trees in the test images.

\section{REFERENCES}

Aksoy, S., Yalniz, I. Z. and Tasdemir, K., 2012. Automatic detection and segmentation of orchards using very high resolution imagery. IEEE Transactions on Geoscience and Remote Sensing, 50(8), pp. 3117-3131.

Bazi, Y., Al-Sharari, H. and Melgani, F., 2009. An automatic method for counting olive trees in very high spatial remote sensing images. In: Proceedings of the 2009 IEEE International Geoscience and Remote Sensing Symposium, Vol. 2, IEEE, Cape Town, South Africa, pp. II.125-II.128.

Du, Y., Chang, C.-I., Ren, H., Chang, C.-C., Jensen, J. O. and DAmico, F. M., 2004. New hyperspectral discrimination measure for spectral characterization. Optical Engineering, 43(8), pp. 1777-1786.

Erickson, M., 2004. Segmentation and Classification of Individual Tree Crowns in High Spatial Resolution Aerial Images. $\mathrm{PhD}$ thesis, Swedish University of Agricultural Sciences, Uppsala.

FAO, F., 2012. Statistical Yearbook 2013: World Food and Agriculture. FAO (Food and Agriculture Organization of the United Nations), Rome.

Gomes, M. and Maillard, P., 2014. Integration of a marked point process and template matching for the identification of individual tree crowns in an urban and a wooded savanna environment in Brazil. In: Proceedings of SPIE Remote Sensing 2014, Vol. 9245, International Society for Optics and Photonics, Amsterdam, The Netherlands, pp. 92450X.1 - 92450X.12. 
González, J., Galindo, C., Arevalo, V. and Ambrosio, G., 2007. Applying image analysis and probabilistic techniques for counting olive trees in high-resolution satellite images. In: Advanced Concepts for Intelligent Vision Systems, Springer, pp. 920-931.

Ke, Y. and Quackenbush, L. J., 2011. A review of methods for automatic individual tree-crown detection and delineation from passive remote sensing. International Journal of Remote Sensing, 32, pp. 4725-4747.

Larsen, M., Eriksson, M., Descombes, X., Perrin, G., Brandtberg, T. and Gougeon, F., 2011. Comparison of six individual tree crown detection algorithms evaluated under varying forest conditions. International Journal of Remote Sensing, 32(20), pp. 5827-5852.

Perrin, G., 2006. Etude du couvert forestier par processus ponctuels marqués. $\mathrm{PhD}$ thesis, Ecole Centrale Paris.

Pu, R. and Landry, S., 2012. A comparative analysis of high spatial resolution IKONOS and WorldView-2 imagery for mapping urban tree species. Remote Sensing of Environment, 124, pp. 516-533.

Recio, J., Hermosilla, T., Ruiz, L. and J.Palomar, 2013. Automated extraction of tree and plot-based parameters in citrus orchards from aerial images. Computers and Electronics in Agriculture 90, pp. 24-34.

Santoro, F., Tarantino, E., Figorito, B., Gualano, S. and D'Onghia, A. M., 2013. A tree counting algorithm for precision agriculture tasks. International Journal of Digital Earth, 6(1), pp. 94-102.

Shao, G. and Reynolds, K. M., 2006. Computer applications in sustainable forest management,. Vol. 11, Springer.

Srestasathiern, P. and Rakwatin, P., 2014. Oil palm tree detection with high resolution multi-spectral satellite imagery. Remote Sensing (MDPI), 6(10), pp. 9749-9774.

Stuckens, J., Somers, B., Albrigo, G. L., Dzikiti, S., Verstraeten, W. W., Swennen, R., Verreyne, S. and Coppin, P., 2010. Offnadir viewing for reducing spectral mixture issues in citrus orchards. Photogrammetric Engineering \& Remote Sensing, 76(11), pp. 1261-1274.

Tasdemir, K., 2010. Classification of hazelnut orchards by selforganizing maps. In: IAPR Workshop on Pattern Recognition in Remote Sensing, 2010, IEEE, pp. 1-4.

Wulder, M., Niemann, K. O. and Goodenough, D. G., 2000. Local maximum filtering for the extraction of tree locations and basal area from high spatial resolution imagery. Remote Sensing of Environment, 73(1), pp. 103-114. 